\title{
BMJ Open A randomised controlled trial to compare the clinical and cost-effectiveness of prism glasses, visual search training and standard care in patients with hemianopia following stroke: a protocol
}

\author{
F J Rowe, ${ }^{1} \mathrm{P}$ G Barton, ${ }^{2} \mathrm{E}$ Bedson, ${ }^{3} \mathrm{R}$ Breen, ${ }^{3} \mathrm{E}$ J Conroy, ${ }^{4} \mathrm{E}$ Cwiklinski, ${ }^{3}$ \\ C Dodridge, ${ }^{5}$ A Drummond, ${ }^{6} \mathrm{M}$ Garcia-Finana, ${ }^{4} \mathrm{C}$ Howard, ${ }^{7} \mathrm{~S}$ Johnson, ${ }^{8}$ \\ C Maclntosh, ${ }^{5} \mathrm{C}$ P Noonan, ${ }^{9}$ A Pollock, ${ }^{10} \mathrm{~J}$ Rockliffe, ${ }^{11} \mathrm{C}$ Sackley, ${ }^{12} \mathrm{~T} \mathrm{Shipman,}^{13}$ \\ VISION (vision impairment in stroke: intervention or not)
}

To cite: Rowe FJ, Barton PG, Bedson $\mathrm{E}$, et al. $\mathrm{A}$ randomised controlled trial to compare the clinical and cost-effectiveness of prism glasses, visual search training and standard care in patients with hemianopia following stroke: a protocol. BMJ Open 2014;4:e005885. doi:10.1136/bmjopen-2014005885

- Prepublication history for this paper is available online. To view these files please visit the journal online (http://dx.doi.org/10.1136/ bmjopen-2014-005885).

Received 7 June 2014 Accepted 25 June 2014

\section{CrossMark}

For numbered affiliations see end of article.

Correspondence to Dr Fiona Rowe; rowef@liverpool.ac.uk

\section{ABSTRACT}

Introduction: Homonymous hemianopia is a common and disabling visual problem after stroke. Currently, prism glasses and visual scanning training are proposed to improve it. The aim of this trial is to determine the effectiveness of these interventions compared to standard care.

Methods and analysis: The trial will be a multicentre three arm individually randomised controlled trial with independent assessment at 6 week 12 week and 26 week post-randomisation. Recruitment will occur in hospital, outpatient and primary care settings in UK hospital trusts. A total of 105 patients with homonymous hemianopia and without ocular motility impairment, visual inattention or pre-existent visual field impairment will be randomised to one of three balanced groups. Randomisation lists will be stratified by site and hemianopia level (partial or complete) and created using simple block randomisation by an independent statistician. Allocations will be disclosed to patients by the treating clinician, maintaining blinding for outcome assessment. The primary outcome will be change in visual field assessment from baseline to 26 weeks. Secondary measures will include the Rivermead Mobility Index, Visual Function Questionnaire 25/10, Nottingham Extended Activities of Daily Living, Euro Qual-5D and Short Form-12 questionnaires. Analysis will be by intention to treat.

Ethics and dissemination: This study has been developed and supported by the UK Stroke Research Network Clinical Studies Group working with service users. Multicentre ethical approval was obtained through the North West 6 Research ethics committee (Reference 10/H1003/119). The trial is funded by the UK Stroke Association. Trial Registration: Current Controlled Trials ISRCTN05956042. Dissemination will consider usual scholarly options of conference presentation and journal publication in addition to patient and public dissemination with lay summaries and articles.

Trial Registration: Current Controlled Trials ISRCTN05956042.

\section{INTRODUCTION}

Homonymous hemianopia results in loss of one-half of the visual field in both eyes. ${ }^{1}{ }^{2}$ The reported prevalence of visual field loss following stroke has been as high as $63 \%^{3}$ in hospital populations although estimates vary widely as the proportion testing positive is highly dependent on time post-stroke. Homonymous hemianopia on admission is linked to poor early survival and, conversely, around $10 \%^{3}$ experience full spontaneous recovery within the first 2 weeks. Visual field defects can seriously impact on functional ability and quality of life following stroke. ${ }^{45}$ Patients with visual field defects have an increased risk of falling, ${ }^{6}$ impaired ability to read, poor mood and institutionalisation. ${ }^{6-9}$ Visual field loss may impact on a patient's ability to participate in rehabilitation, and may ultimately result in poor long-term recovery. ${ }^{8}$ Visual field loss can result in accidents or injuries which have subsequent cost implications to the National Health Service and the patient. ${ }^{10}$

Service provision is presently inconsistent. $^{11}$ A recent survey of orthoptists has shown that $45 \%$ of stroke services provided no formal vision assessment for patients with stroke. ${ }^{12}$ A further survey of current practice 
in stroke care in patient settings in Scotland identified that only $7 \%$ of stroke units had a policy relating to vision assessment and management. ${ }^{13}$ Both surveys illustrate the lack of standardisation of practice in relation to visual impairment assessment and treatment for stroke survivors. The Department of Health National Stroke Strategy, ${ }^{14}$ argues that vision and visual perceptual difficulties are components requiring multifaceted stroke specific rehabilitation and support. The Royal College of Physicians recently revised their guidance for stroke and currently recommend that every patient who has had a stroke should have a practical assessment of vision and examination of the visual field. ${ }^{15}$ They also advise that any patient whose visual field defect causes practical problems should be taught compensatory techniques with prism treatment provided only by those with expertise in this treatment. The Scottish Intercollegiate Guideline Network (SIGN) guidelines for management of patient with stroke (SIGN 118) ${ }^{16} 17$ advocates that all patients with stroke should be screened for visual problems and also suggests visual scanning compensatory training techniques as potentially improving function outcomes after stroke. The SIGN 118 guidelines also stress the need for further high-quality research to investigate the effectiveness of interventions for visual field defects.

Two key interventions commonly used in the clinical setting to improve vision in hemianopia are visual scanning compensatory training and provision of prisms. ${ }^{13}$ We completed a literature search and identified a number of reviews, which have investigated the efficacy of interventions for hemianopia after stroke. ${ }^{8-22}$ We extracted data on any intervention studies investigating visual scanning compensatory training or prisms and searched for any subsequent or on-going randomised controlled trials (RCTs).

A number of intervention studies have been conducted for visual scanning compensatory training. There is consistency between these studies in identifying benefits associated with scanning or eye movement training. One RCT has reported on the use of either 15 dioptre plastic press-on fresnel prisms or no prism treatment. The results of this study demonstrated improvements in visual impairment, but not in functional outcomes. ${ }^{23}$ It was limited by the inclusion of patients with hemianopia and neglect, by the small prism power and by use of screening vision measures. We identified four longitudinal studies of prism use. Bowers $e t a l^{24}$ and Giorgi et $a l^{25}$ completed prospective cohort studies, investigating Fresnel prism peripheral segments. The reported outcome for these studies was 'clinical success', as measured by continued wear: $74 \%$ and $67 \%$ of participants, respectively, were reported to continue to wear the prisms at the end of the study, and $47 \%$ and $42 \%$, respectively, at long-term follow-up. In a study of 12 patients fitted with 40 dioptre prisms, an effective expansion of the visual field (in the presence of prism wear only) was reported of up to $20^{\circ 26}$; however, there was no control group included in this study.
Our aim is to undertake a pilot RCT to provide essential information for the conduct of a subsequent full phase III study to determine the clinical and costeffectiveness of visual rehabilitation interventions as compared to NHS standard care, in patients with hemianopia following stroke. We wish to determine whether visual rehabilitation is more effective than standard care (advice only) at improving functional outcome in patients with hemianopia following stroke, and whether prism therapy or visual search therapy is more effective at improving functional outcome in patients with hemianopia following stroke.

\section{METHODS AND ANALYSIS}

This trial is a randomised controlled, multicentre trial. Treatment with Fresnel prisms and treatment with visual search strategies are compared to a control group of standard care (advice only).The template for intervention description and replication (TIDieR) checklist and guide will be used when reporting the results of this trial.

\section{Primary end point}

Change of visual field over time-from baseline to 26 weeks post baseline assessment. Visual field will be measured in degrees.

\section{Secondary end points}

Changes of outcomes A-F (see below) over time, from baseline up to 26 weeks post baseline assessment.

A. Visual function questionnaire (VFQ 25-10): change in perceived ability relating to activities of daily living

B. Rivermead Mobility Index: change in functional mobility

C. Nottingham extended activities of daily living (NEADL): change in instrumental activities

D. EQ-5D: change in health-related quality of life

E. SF-12: change of general health status

F. Radner test: change of reading speed and accuracy

\section{Inclusion criteria}

Patients with the following characteristics will be eligible for inclusion in the trial:

A. 18 years of age or older;

B. Best corrected visual acuity of 0.5 or better in each eye (left and right separately) at distance;

C. Stable homonymous hemianopia (partial or complete) induced by stroke occurring more than 2 weeks ago but less than 26 weeks ago;

D. Refractive error within \pm 5 dioptres;

E. Willing and able to give consent for the study;

F. Prior to stroke able to read and understand English.

Vision measures will be established by orthoptic assessment. Stroke will be defined using WHO guidelines: 'occurrence of rapidly developing signs of focal or global disturbance of cerebral function, lasting longer than $24 \mathrm{~h}$ or leading to death, with no apparent cause other than that of vascular origin.' 


\section{Exclusion criteria}

All patients who do not meet the inclusion criteria listed above will be excluded from the trial. Otherwise, patients with the following characteristics will be excluded from the trial:

A. Inability to consent due to severe cognitive impairment;

B. Ocular motility impairment and/or visual inattention in addition to the visual field impairment (as assessed by the orthoptist—saccadic impairment introduced by the hemianopia is not an exclusion criteria)

C. Pre-existent visual field impairment due to previous stroke.

We will not include patients who are recruited to other trials or studies that treat visual problems.

\section{Recruitment}

Each week the Orthoptist will contact the admitting ward (usually a stroke unit) to identify those patients recently admitted with a stroke and suspected of having homonymous hemianopia. The Orthoptist will liaise with the stroke Research Nurse to identify these patients and arrange appointments. All identified patients will be contacted either in person if an in patient, or via telephone for those who have been discharged. Patients will be invited for eye assessment, conducted by the Orthoptist in the eye clinic. Patients will be provided with information sheets with full explanation of the trial before obtaining informed written consent. Where the patient is unable to write but is able to indicate consent, witnessed consent will be obtained (figure 1).

All the patients who undergo screening will be recorded on a 'Screening Log' regardless of whether they decide to participate in the trial. Reasons patients are not randomised, for example, not eligible will be recorded. Reasons for declining to participate will be asked routinely but it will be made clear that they do not have to provide a reason unless happy to do so.

\section{Enrolment/baseline}

Once informed consent has been obtained from the patient, the Orthoptist and Research Nurse will use the baseline case report form to collect baseline characteristics including patient demographics, visual signs and symptoms, visual acuity measures, any additional ocular problems or comorbidity, severity of stroke and level of disability. The eligibility case report form will be completed by the Orthoptist. Patient completed questionnaires will also be collected.

Randomisation of a participant will take place once:

1. Eligibility assessment has been fulfilled;

2. Fully informed written consent has been obtained;

3. Baseline assessments, including visual field assessment and the degree of disability have been completed.

Participants will be randomised using a secure $(24 \mathrm{~h})$ web-based randomisation programme controlled centrally by the Clinical Trials Research Centre. Participant treatment allocation will be displayed on a secure webpage and an automated email confirmation sent to the authorised randomiser, the Principal Investigator (PI) and the trial coordinator. In case of failure of this system, pre-prepared back up envelopes will be available. It is the responsibility of the PI or delegated research staff to ensure that sites have surplus randomisation lists and back up envelopes throughout the recruitment period to prevent any delays in the randomisation process.

\section{Method of randomisation}

Participants will be individually randomised to the three groups of equal size: treatment with prism therapy; treatment with visual search training; or standard care (advice only). Randomisation lists, stratified by centre and degree of hemianopia (partial or complete), will be produced by an independent statistician at the CTRC using simple block randomisation. Outcome assessors will remain blind to participant intervention. However, due to the nature of the intervention, it is not possible to blind other study personnel or the participant.

\section{Interventions}

Treatment with the allocated intervention will start immediately following randomisation.

\section{Arm A: treatment with prism therapy}

All participants in this group will be given sector Fresnel prisms of 40 prism dioptre strength on their glasses (or plain glasses if not already worn) which displaces images away from the affected side. ${ }^{26}$ Prisms are not placed over central vision as this would cause double vision and would therefore pose a risk for participant safety. Consequently, by avoiding central vision, there are limited side effects or risks to use of these prisms. Separate prism segments are used to expand the upper and lower quadrants. The prism shifts objects providing a real field of view expansion of degree comparable to the prism power used, for example, $20^{\circ}$ with 40 prism dioptre. This results in 'confusion' or overlap of real and shifted objects.

If the participant wears bifocals or varifocals the prisms will be affixed to alternative single lens/distance glasses or plain glasses. If this is not possible only the upper prism will be affixed to the participants bifocalsor varifocals. This will be captured on the case report forms. Participants will require one Fresnel prism, which is cut to fit the upper and lower segment of one lens of their glasses. The cutting and fitting of the prism are undertaken by a specialist Orthoptist who will ensure that central gaze is not disrupted. Prior to fitting the prism will be inspected for any faults by the Orthoptist. The participant will be instructed to maintain central fixation through their glasses. They will then be instructed to use head movements to explore their field to the affected side when they become aware of an 
Figure 1 Flow chart for trail.

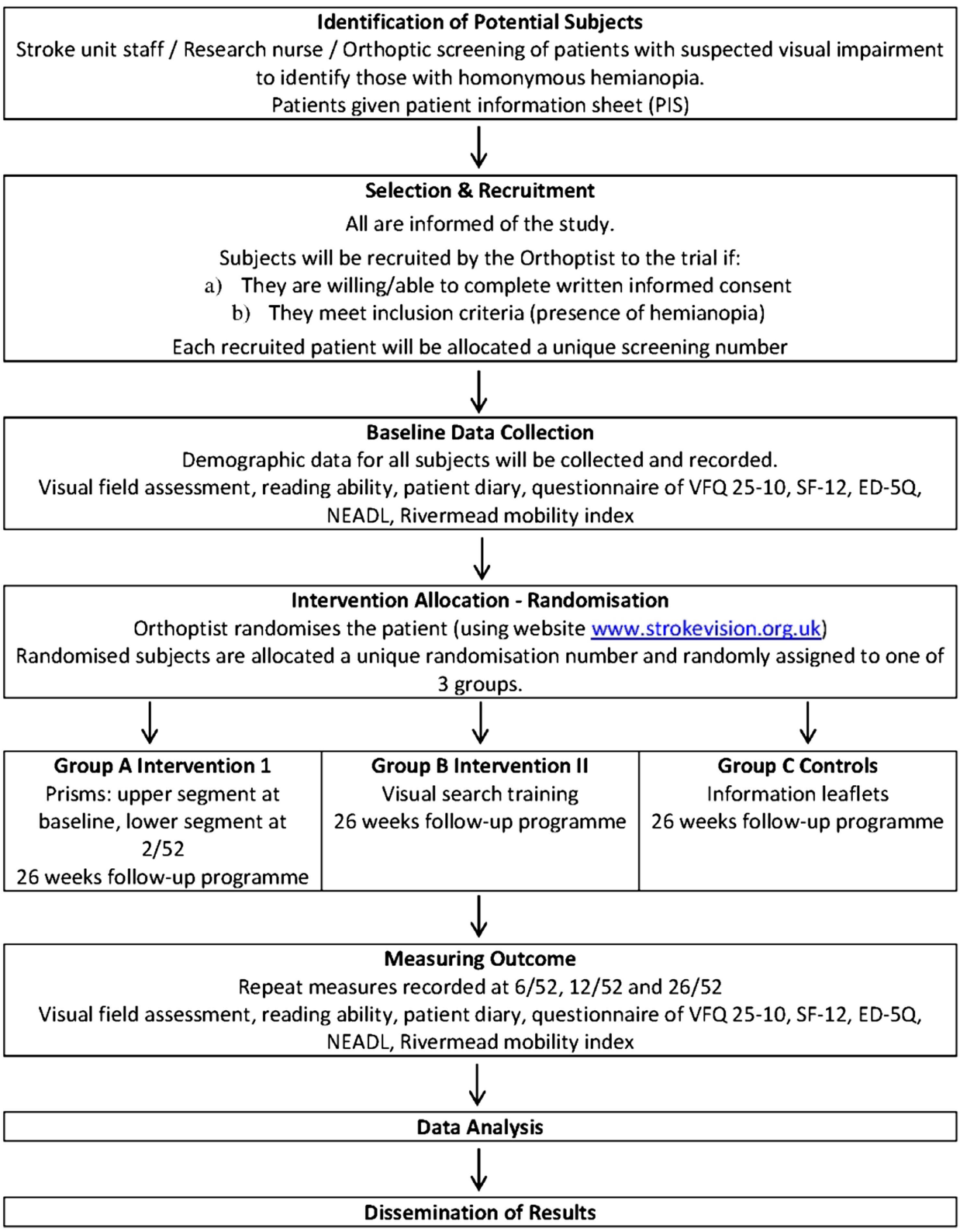

object of interest through the prism. The first prism will be placed at the participant's first visit; if possible the second prism will also be fitted at this time. However, if the participant is not comfortable with both prisms being fitted at once, the second prism can be placed at a second visit ( 2 weeks later, \pm 1 week) if no adaptation difficulties to the first prism have occurred. If adaption difficulties have occurred the patient can continue with only the first prism and this will be captured on the case report forms.

The prisms should be worn for a minimum of $2 \mathrm{~h}$ daily from prism affixation until 6-week follow-up visit as a minimum, after this the patient can elect to continue treatment if they wish. This information will be captured on the case report forms and also the participant completed diaries will record whether the glasses were worn for $2 \mathrm{~h}$. This group will also receive the same information leaflets as described for Arm C.

\section{Arm B: treatment with visual search therapy}

All participants in this group will be given visual search training. This comprises an A4landscape card with horizontal and diagonal numbered circles radiating out from a central fixation target. The participant will be instructed to hold this at a distance of 8 inches from their eyes (to ensure a wide field of vision is utilised), glasses can be worn as required. Participants will be asked to transfer gaze quickly between printed targets on the A4 card. The targets are printed off centre to the right and left sides along the horizontal as well as oblique planes to ensure stimulation of a wide area in the blind and seeing parts of the visual field. 
Participants will be instructed to continually scan between the various targets for $30 \mathrm{~min}$ daily from baseline until their 6-week follow-up, after which they can elect to continue treatment if they wish. This information will be captured on the case report forms and also the participant completed diaries will record whether the exercises were performed for $30 \mathrm{~min}$ continuously or in blocks. Participants will be instructed on the scanning exercises following randomisation to ensure their understanding of the procedure of doing this training. In addition, printed instructions will be provided with the visual training target card. This group will also receive the same information leaflets as described for Arm C.

\section{Arm C: control-standard care (advice only)}

At time points according to local policy, the participant will be given information leaflets from the Stroke Association and the Royal National Institute for the Blind (RNIB) about visual impairment following stroke.

All three arms will receive the same information leaflets.

\section{Assessment of compliance with trial treatments}

Compliance with treatment up to the first follow-up appointment at 6 weeks is important and participants and carers will be advised of the importance of continued treatment during this early time period.

Each participant will have a diary to record their comments in relation to treatment and whether prisms, visual search training or leaflets have been found beneficial or not. Usage of the prisms will be measured by recording the continued wear of the prisms (taken from the participant's diary) and participants receiving the visual search strategy treatment will be asked to keep a diary of the time during each day when treatment was undertaken and the duration.

The PI or research nurse will explain how to complete the diaries before the participant leaves hospital. Participants will be instructed to bring back the diary to their follow-up visits.

\section{Bias protection}

Baseline assessments and randomisation will be conducted prior to knowledge of allocation. Patient compliance to group allocation will be followed up by investigators to ensure that the treatment randomised is given.

The chief investigator is masked to the randomisation process and treatment groups. It is clearly not possible to mask the local investigators (principal investigators and research nurses) responsible for treating the participants.

\section{Treatment modifications}

The participant will maintain their treatment allocation until their 6-week follow-up visit. The participant can then elect to continue treatment and this decision will be captured on the follow-up visit case report forms and also in the participant completed diaries. At the end of the 26-week follow-up period the participant may continue their treatment as per local policy and for control participants, may opt to undertake one of the trial treatments if indicated and appropriate for them at that time.

\section{Schedule for follow-up}

The expected follow-up of each participant is 26 weeks with follow-up assessment at 6 week, 12 week and 26 week post-start of treatment. One-week windows $( \pm 1$ week) are allowable on the 6-week visit, while 2-week windows ( \pm 2 weeks) are allowable for the 12-week and 26-week visits. Where possible, appointments will be booked at the start of the follow-up visit window so that if a participant does not or cannot attend their appointment, a further appointment can be remade within the window. The participant will be contacted directly regarding any failed appointment attendance. All visits will be conducted in out-patient eye clinic departments due to the location of visual field perimeters in eye clinics. Participants withdrawn from trial treatment will be asked to continue with trial follow-up and attend the follow-up visits. If a participant does not wish to continue in the trial, a Consent Withdrawal case report form will be completed to capture the date and reason for trial withdrawal. A minimum of the primary outcome data will be collected for participants who withdraw from trial treatment or for protocol deviations.

\section{Procedures for assessing efficacy}

Efficacy of the trial treatments will be measured throughout the period of the trial using objective and subjective measures.

Baseline characteristics will include sex, date of birth, date of stroke onset, type of stroke, location of stroke, Barthel score and visual symptoms. Gold standard visual field assessment will consist of evaluation of the visual field in either eye as well as a binocular Esterman field of vision using Humphrey or Octopus automated perimetry and Goldmann manual perimetry with standard fixation monitoring strategies of fixation loss, false-positive and false-negative responses. Outcome measures will be taken at the same time points of baseline, 6 week, 12 week and 26 week follow-up.

\section{Visual field assessment}

The visual field assessment will be conducted by a qualified Orthoptist at baseline and at the 6-week, 12-week and 26-week follow-up visits. An Esterman strategy is to be used for quantitative visual field assessment. This can be performed using either:

- The Esterman programme on Humphrey or Octopus perimetry;

- The III4e target on Goldmann with additional checks of static points in the central visual field. 
A template for Goldmann perimetry will be supplied for standardisation which will match to the Esterman strategy on Humphrey and Octopus perimetry. A binocular visual field will be measured first followed by monocular assessment of the right and left eyes. Where it is not possible to use either of these methods then the standardised confrontation method will be used. Whichever method used at baseline must be duplicated at the patient's follow-up visits. Where the confrontation method is used at baseline one of the above quantitative methods must be used at the follow-up if possible in addition to repeating the confrontation method.

\section{Reading ability}

Reading ability will be assessed by a qualified Orthoptist at baseline and at the 6-week, 12-week and 26-week follow-up visits using the Radner reading test.

\section{Participant completed outcome measures}

At baseline and the 6-week, 12-week and 26-week follow-up visits the participant will be asked to complete a questionnaire booklet containing the following outcome measures:

- Visual function questionnaire (VFQ 25-10)

- Rivermead Mobility Index

- Nottingham extended activities of daily living assessment (NEADL)

- ED-5Q

- Short Form-12 (SF-12)

All measures have been used extensively in previous stroke research and are sensitive to change, valid and reliable. Inclusion of each measure has been fully discussed and approved by the collaborative team and user group involved in preparing the proposal for this trial. Completion of the questionnaire booklets will ideally precede any discussion with the PI or delegated other. The PI or delegated other will ensure all questions have been answered and that the randomisation number and time point at which the booklet was administered are recorded on the booklet. If the participant requires help when completing the forms, because of difficulties with writing, reading or aphasia, this will be recorded by the PI.

Participants must complete the baseline booklet before treatment allocation has been revealed. If the participant is too unwell to receive the questionnaire booklet or has missed at time point the research nurse must inform the Clinical Trials Research Centre.

\section{Sample size}

There is little published on the recovery of visual field following stroke, and therefore, insufficient data are available to carry out a formal power calculation. The proposed trial is designed to provide information for a phase III trial to determine the effectiveness of visual rehabilitation (prism glasses or visual search training) compared to the standard care intervention in patients with hemianopia following stroke.
We consider a sample of 30 participants per group will obtain precise estimates of the parameters that are required for the sample size calculation ${ }^{27}$ (variance of visual field per group, within-patient correlation-this is the correlation between observations made at several time points and loss to follow-up rates) as well as to generate sufficient data on recruitment rates, compliance of practitioners with randomisation and user views. Additionally we increased the sample size needed per group to 35 participants to allow for up to $15 \%$ drop out from the study (based on stroke rehabilitation literature) with the reasons for drop out being recorded (ie, a total of 105 participants).

\section{Analysis plan}

The trial will be analysed and reported according to the 'Consolidated Standard of Reporting Trials' (CONSORT), TIDieR and the International Conference on Harmonisation E9 guidelines. No formal statistical interim analysis of the outcome data is planned. Interim monitoring will focus on recruitment, safety and exploratory analysis to ensure baseline characteristics are balanced between groups.

Data will be accessed by the trial team. A full and detailed statistical analysis plan will be developed prior to the final analysis of the trial. The main features of the statistical analysis plan are included here. Outcome analysis will be performed following an intention-to-treat approach. The data collected will be used to estimate the sample size required for a phase III trial based on the analysis of covariance approach. ${ }^{28}$ Additionally, we will apply a longitudinal linear mixed effects model to explore changes in visual field over time. A p value of 0.05 (5\% level) will be used to declare statistical significant and 95\% CIs of the estimated effects will be reported. Rather than adjust for multiplicity relevant results from other studies already reported in the literature will be taken into account in the interpretation of results. Analysis will be undertaken to ensure that the assumptions underlying the modelling techniques used are met. As this trial is a pilot trial to establish sample size parameters required for a full study and no adjustment for multiplicity, all analyses will be treated as exploratory and interpreted with caution.

\section{ETHICAL CONSIDERATIONS}

The study will abide by the principles of the World Medical Association Declaration of Helsinki (1964) and the Tokyo (1975), Venice (1983), Hong Kong (1989) and South Africa (1996).

The trial protocol has been approved by the Health Research Authority Multicentre Research Ethics Committee (MREC) and by independent review at the Research \& Development (R\&D) offices at participating sites (Reference 10/H1003/119). All participants are required to provide informed, written consent. 
Trial monitoring and oversight

Trial monitoring will be informed by the VISION risk assessment and will be conducted as per the VISION Trial Monitoring Plan to ensure that the rights and wellbeing of human participants are protected during the course of the clinical trial and that the data are credible and accurate.

Data capture will be in the form of paper copies that will be returned as an on-going process from each site to the CTRC within 7 days of completion. All completed forms for each patient will be expected within 7 days of final follow-up visit at 26 weeks.

The trial case report form (CRF) is the primary data collection instrument for the trial. All data requested on the CRF must be recorded and all missing data must be identified and queried. CRFs will be photocopied at site and the originals returned to the CTRC within 7 days of the time specified for completion, unless stated otherwise (photocopies of completed CRFs will be kept in the site file).

Participants will complete the following questionnaires at specified intervals (baseline and 6 week, 12 week and 26 week follow-up visits):

- VFQ 25-10

- EQ-5D

- SF-12

- NEADL

- Rivermead Mobility Index

- Treatment diary

The participant initials and randomisation number will be clearly labelled on all these documents. Research staff will also complete the relevant 'centre use' section on the front cover of each questionnaire or diary. Diaries and questionnaires will be returned to CTRC alongside the relevant CRF treatment pages where possible.

Data electronically stored at CTRC will be checked for missing or unusual values (range checks) and checked for consistency within participants. The trial monitoring plan will detail methods in place to ensure reliability and validity of the trial data. Any data that breaches these checks will be returned to the site in the form of data queries. Data query forms will be produced at the CTRC from the trial database and sent either electronically or through the post to a named individual (as listed on the site delegation $\log$ ). Sites will respond to the queries providing an explanation/resolution to the discrepancies and return the data query forms to CTRC. The forms will then be filed along with the appropriate CRFs and the appropriate corrections made on the database.

In order to perform their role effectively, the trial coordinator (or monitor) and persons involved in quality assurance and audit/inspection may need direct access to primary data, for example, patient records, laboratory reports, appointment books, etc. Since this affects the patient's confidentiality, this fact is included on the Patient Information Sheet and Informed Consent Form.
A Trial Management Group (TMG) will be formed comprising the Chief Investigator, other lead investigators (statistical, clinical and non-clinical) and members of the CTRC. The TMG will be responsible for the day-to-day running and management of the trial and will meet approximately three times a year. A quorum of three group members will be required to conduct a TMG meeting.

The independent Data and Safety Monitoring Committee (IDSMC) will consist of an independent chairperson plus two independent members including a statistician and clinician. The IDSMC will be responsible for reviewing and assessing recruitment, interim monitoring of safety and effectiveness, trial conduct and external data. The IDSMC will first convene prior to the start of recruitment and will then define frequency of subsequent meetings (at least annually). The IDSMC will provide a recommendation to the Trial Steering Committee concerning the continuation of the study.

The Trial Steering Committee will oversee the trial. Independent members will consist of an independent chairperson, independent experts in the field of visual impairment following stroke and a biostatistician. Non-independent members will consist of the local Investigators. The role of the TSC is to provide overall supervision for the trial and provide advice through its independent Chairman. The ultimate decision for the continuation of the trial lies with the TSC.

\section{Patient and public involvement}

This trial has involved a stroke survivor directly in the development of this protocol (JR) and will liaise closely with her for advice and direction throughout the conduct of the trial and in the dissemination process. Involvement of stroke survivors in oversight committees is also planned for this trial.

\section{DISCUSSION}

A recent Cochrane systematic review reported three main approaches to visual rehabilitation: substitution, adaptation or restitution. ${ }^{29}$ Following a comparison of published data, the authors stated that generalisable conclusions could not be reached regarding the use of any approach to visual rehabilitation for homonymous hemianopia because there was insufficient high-quality evidence. Based on limited evidence, it was indicated that the greatest improvement in function, but not visual field defects, followed visual search training. It was recommended that future RCTs should determine the effect of compensatory scanning training OR restitutive interventions OR substitutive interventions compared to no treatment, control or placebo. ${ }^{29}$

There are no large scale RCTs to establish the effectiveness of prisms versus visual scanning training versus no intervention. This trial will explore the effectiveness of prism therapy (using base-out Fresnel prisms)and visual search therapy (using paper-based visual scanning 
training) versus standard care (advice only with no intervention). Outcome measures will include objective measurement of visual fields and reading plus functional measures by health-related and vision-related questionnaires of daily living.

This trial is designed as a pilot randomised controlled trial to consider issues of recruitment and consent, retention rates during the trial, compliance and adherence with the interventions, appropriateness of blinding procedures and randomisation, acceptability of the interventions and trial procedures to the participants and investigators, appropriateness of outcome measurements and logistics of multicentre procedures. The results of the trial will provide information that could help to improve allocation of treatment and resources in visual rehabilitation post-stroke. This trial is funded by the UK Stroke Association, is sponsored by the University of Liverpool, has received multicentre ethical approval through the North West 6 Research Ethics Committee (Reference 10/H1003/119) and trial registration for current controlled trials (ISRCTN05956042).

\section{Author affiliations}

${ }^{1}$ Department of Health Services Research, University of Liverpool, Liverpool, UK

${ }^{2}$ Department of Elderly Care, Warrington and Halton Hospitals NHS

Foundation Trust, Warrington, UK

${ }^{3}$ Clinical Trials Research Unit, University of Liverpool, Liverpool, UK

${ }^{4}$ Department of Biostatistics, University of Liverpool, Liverpool, UK

${ }^{5}$ Department of Orthoptics, Oxford University Hospitals NHS Trust, Oxford, UK

${ }^{6}$ School of Health Sciences, University of Nottingham, Nottingham, UK

${ }^{7}$ Department of Orthoptics, Salford Royal NHS Foundation Trust, Manchester, UK

${ }^{8}$ Eye Clinic Impact Team, Royal National Institute for the Blind, Birmingham, UK

${ }^{9}$ Department of Ophthalmology, Aintree University Hospital NHS Foundation Trust, Liverpool, UK

${ }^{10}$ Nursing, Midwifery and Allied Health Professions Research Unit, Glasgow

Caledonian University, Glasgow, UK

${ }^{11}$ Speakability North West, London, UK

${ }^{12}$ Faculty of Medicine and Health, University of East Anglia, Norwich, UK

${ }^{13}$ Department of Orthoptics, Sheffield Teaching Hospitals NHS Foundation Trust, Sheffield, UK

Contributors FJR, PGB, RB, AD, MG-F, SJ, CM, CPN, AP, JR and CS conceived of the study, participated in the design and coordination and helped to draft the manuscript. EB, CD, CH and TS participated in the coordination and helped to draft the manuscript. MG-F supervised the statistical analysis. BC performed the statistical analysis, data monitoring and helped to draft the manuscript. EC participated in the coordination, performed data entry and helped to draft the manuscript. All authors read and approved the final manuscript

Funding This trial was funded by the UK Stroke Association (TSA 2010/02).

Competing interests EC was paid a salary by the UK Stroke Association.

Provenance and peer review Not commissioned; peer reviewed for ethical and funding approval prior to submission.

Open Access This is an Open Access article distributed in accordance with the Creative Commons Attribution Non Commercial (CC BY-NC 4.0) license, which permits others to distribute, remix, adapt, build upon this work noncommercially, and license their derivative works on different terms, provided the original work is properly cited and the use is non-commercial. See: http:// creativecommons.org/licenses/by-nc/4.0/

\section{REFERENCES}

1. Rowe FJ, VIS group. Visual impairment following stroke. Do stroke patients require vision assessment? Age Ageing 2009;38:188-93.

2. Kerkhoff G. Restorative and compensatory therapy approaches in cerebral blindness-a review. Restor Neurol Neurosci 1999;15:255-71.

3. Gray CS, French JM, Bates D, et al. Recovery of visual fields in acute stroke: homonymous hemianopia associated with adverse prognosis. Age Ageing 1989;18:419-21.

4. Jongbloed L. Prediction of function after stroke: a critical review. Stroke 1986;17:765-76.

5. Dombovy ML, Sandok BA, Basford JR. Rehabilitation for stroke: a review. Stroke 1986;17:363-9.

6. Ramrattan RS, Wolfs RC, Panda-Jonas S, et al. Prevalence and causes of visual field loss in the elderly and associations with impairment in daily functioning: the Rotterdam Study. Arch Ophthalmol 2001;119:1788-94.

7. Kaplan J, Hier DB. Visuospatial deficits after right hemisphere stroke. Am J Occup Ther 1982;36:314-21.

8. Jones SA, Shinton RA. Improving outcome in stroke patients with visual problems. Age Ageing 2006;35:560-5.

9. Windsor RL, Windsor LK. Hemianopsia: loss of half of the visual field after stroke or traumatic brain injury. http://www.lowvision.org/ understanding\%20hemianopsia1.htm (accessed 23 Mar 2014).

10. Black A, Wood J. Vision and falls. Clin Exp Optom 2005; 88:212-22.

11. Rowe FJ. Reply. Letters to the Editor. Age Ageing 2009;38:630-1.

12. Rowe FJ. Who sees visual impairment following stroke? Strabismus 2010;18:37-40.

13. Pollock A, Hazelton C, Brady M. Visual problems after stroke: a survey of current practice by occupational therapists working in UK stroke inpatient settings. Top Stroke Rehabil 2011;18:643-53.

14. Department of Health. National stroke strategy. London: DH, December 2007

15. Royal College of Physicians Intercollegiate Stroke Working Party. National clinical guidelines for stroke. 3rd edn. London: Royal College of Physicians, 2008.

16. Scottish Intercollegiate Guidelines Network. Management of patients with stroke. Rehabilitation, prevention and management of complications and discharge planning. Guideline number 64 November 2002.

17. Pollock A, Chung C; on behalf of SIGN 64 Update Committee. Recommendations of visual problems after stroke: development of new SIGN guidelines (abstract). Int $J$ Stroke 2009; 4(Suppl 2):8-9.

18. Pambakian AL, Mannan SK, Hodgson TL, et al. Saccadic visual search training: a treatment for patients with homonymous hemianopia. J Neurol Neurosurg Psychiatry. 2004; 75:1443-8.

19. Bouwmeester L, Heutink J, Lucas $C$. The effect of visual training for patients with visual field defects due to brain damage: a systematic review. J Neurol Neurosurg Psychiatry 2007;78:555-64.

20. Riggs RV, Andrews $\mathrm{K}$, Roberts $\mathrm{P}$, et al. Visual deficit interventions in adult stroke and brain injury. Am J Phys Med Rehabil 2007;86:853-60.

21. Barrett BT. A critical evaluation of the evidence supporting the practice of behavioural vision therapy. Ophthalmic Physiol Optics 2009;29:4-25.

22. Schofield TM, Leff AP. Rehabilitation of hemianopia. Curr Opin Neurol 2009;22:36-40.

23. Rossi PW, Kheyfets S, Reding MJ. Fresnel prisms improve visual perception in stroke patients with homonymous hemianopia or unilateral visual neglect. Neurology 1990;40:1597-9.

24. Bowers AR, Keeney K, Peli E. Community-based trial of a peripheral prism visual field expansion device for hemianopia. Arch Ophthalmol 2008;126:657-64.

25. Giorgi RG, Woods RI, Peli E. Clinical and laboratory evaluation of peripheral prism glasses for hemianopia. Optom Vision $\mathrm{Sci}$ 2009;86:492-502.

26. Peli E. Field expansion for homonymous hemianopia by optically induced peripheral exotropia. Optom Vision Sci 2000;77:453-64.

27. Lancaster G, Dodd S, Williamson PR. Design and analysis of pilot studies: recommendations for good practice. J Eval Clin Pract 2004;10:307-12.

28. Machin D, Campbell M, Fayers $\mathrm{P}$, et al. Sample size tables for clinical studies. 2nd edn. Blackwell Science, 1997.

29. Pollock A, Hazelton C, Henderson CA, et al. Interventions for visual field defects in patients with stroke. Cochrane Database Syst Rev 2011;(10):CD008388. Review. 